\title{
ATUAÇÃO DOS AGENTES COMUNITÁRIOS DE SAÚDE NA ESTRATÉGIA SAÚDE DA FAMÍLIA
}

Elaine Regina Prudencio da Silva', Luiza Helena de Oliveira Cazola², Maria de Fátima Meinberg Cheade², Renata Palópoli Pícoli ${ }^{4}$

RESUMO: Este estudo teve como objetivo caracterizar a atuação dos Agentes Comunitários de Saúde de Unidades Básicas de Saúde da Família de Três Lagoas - Mato Grosso do Sul. Trata-se de um estudo descritivo e de abordagem quantitativa realizado com 68 agentes de sete unidades com Estratégia Saúde da Família. As respostas à entrevista individual foram analisadas pela estatística descritiva. Predominaram mulheres entre 40 e 49 anos, casadas, com ensino médio completo e atuando por cinco a dez anos. A maioria participou de curso introdutório e todos realizaram alguma capacitação. Os participantes relataram conhecer suas atribuições e apontaram a visita domiciliar como atividade mais frequente e consideraram que as ações de controle e prevenção de dengue são inerentes a sua atuação; revelaram realizar atividades que não são de sua responsabilidade. Conclui-se que os agentes comunitários apresentam fragilidades em reconhecer suas atribuições no desempenho de suas atividades.

PALAVRAS-CHAVE: Auxiliares de saúde comunitária; Saúde da família; Atenção primária à saúde.

\section{FUNCTIONING OF COMMUNITY HEALTH WORKERS IN THE FAMILY HEALTH STRATEGY}

ABSTRACT: This study aimed to characterize the functioning of the Community Health Workers in Family Primary Care Centers in Três Lagoas, in Mato Grosso do Sul. It is a descriptive study with a quantitative approach undertaken with 68 workers from seven Family Health Strategy Centers. The responses in the individual interviews were analyzed by descriptive statistics. The majority were women, aged between 40 and 49, married, who had completed middle school and had been working in that role for five to ten years. The majority had participated in the introductory course and all had undertaken some training. The participants reported knowing their responsibilities and indicated the home visit as the most frequent activity, and considered that actions for the control and prevention of dengue are inherent to their work; and they revealed that they undertake activities which are not their responsibility. It is concluded that community workers have weaknesses in recognizing their responsibilities in the performance of their activities.

KEYWORDS: Community health assistants; Family health; Primary health care.

\section{ACTUACIÓN DE LOS AGENTES COMUNITARIOS DE SALUD EN LA ESTRATEGIA SALUD DE LA FAMILIA}

RESUMEN: Este estudio tuvo el objetivo de caracterizar la actuación de los Agentes Comunitarios de Salud de Unidades Básicas de Salud de la Familia de Três Lagoas - Mato Grosso do Sul. Es un estudio descriptivo y de abordaje cuantitativo realizado con 68 agentes de siete unidades con estrategia Salud de la Familia. Las respuestas a la entrevista individual fueron analizadas por la estadística descriptiva. Predominaron mujeres entre 40 y 49 años, casadas, con enseñanza media completa y actuando por cinco a diez años. La mayoría participó de curso introductorio y todos realizaron alguna capacitación. Los participantes relataron conocer sus atribuciones y apuntaron la visita domiciliar como la actividad más frecuente y consideraron que las acciones de control y prevención de dengue son inherentes a su actuación; revelaron realizar actividades que no son de su responsabilidad. Se concluye que los agentes comunitarios presentan fragilidades en reconocer sus atribuciones en el desempeño de sus actividades. PALABRAS CLAVE: Auxiliares de salud comunitaria; Salud da Familia; Atención primaria a la salud.

${ }^{1}$ Enfermeira e Coordenadora da Clínica da Mulher da Prefeitura Municipal de Três Lagoas- MS. Especialista em Saúde Pública e em Enfermagem do Trabalho.

${ }^{2}$ Enfermeira. Doutora em Ciências da Saúde. Professora Colaboradora da Universidade Federal do Mato Grosso do Sul - UFMS.

${ }^{3}$ Enfermeira. Doutora em Ciências da Saúde. Professora do Curso de Graduação em Enfermagem da UFMS.

${ }^{4}$ Fonoaudióloga. Doutora em Saúde Pública. Professora e Coordenadora do Programa Interinstitucional de Interação Ensino-ServiçoComunidade do Curso de Graduação em Medicina da Universidade Anhanguera - Uniderp.

Autor correspondente:

Recebido: 04/04/2012

Luiza Helena de Oliveira Cazola

Aprovado: 09/07/2012

Universidade Federal do Mato Grosso do Sul

Rua Cel. Cacildo Arantes, 365 - 79040-452 - Campo Grande-MS-Brasil

E-mail: luizacazola@gmail.com 


\section{INTRODUÇÃO}

O Ministério da Saúde aprovou por meio da Portaria n. 2.488/2011, a Política Nacional de Atenção Básica, estabelecendo que a equipe multiprofissional mínima se constitui de médico, enfermeiro, técnico ou auxiliar em enfermagem, agente comunitário de saúde (ACS), cirurgião-dentista, auxiliar de saúde bucal e o técnico de saúde bucal ${ }^{(1-2)}$. Na Estratégia Saúde da Família (ESF) o trabalho em equipe é um dos elementos fundamentais para que se alcancem os objetivos desse novo modelo assistencial. $\mathrm{O}$ território destinado à atuação dos profissionais é geograficamente delimitado e abrange cerca de 3 mil a 4,5 mil habitantes ${ }^{(2)}$.

$\mathrm{Na}$ equipe multiprofissional, o ACS se destaca por viabilizar o intercâmbio de experiências, especialmente entre os saberes populares de saúde e os conhecimentos médico-científicos. Além disso, esse profissional facilita o fortalecimento do vínculo com a família e a aproximação das ações de saúde ao contexto domiciliar, incrementando a capacidade da população para enfrentar problemas que interferem na saúde individual e coletiva ${ }^{(3)}$. A Lei n. 10.507/2002 estabelece que para exercer sua profissão o ACS deve residir na área de sua atuação e haver concluído o ensino fundamental e o curso de qualificação básica (curso introdutório) para a atividade ${ }^{(4)}$. Posteriormente, diretrizes para acesso à carreira e regulamentação da atividade de ACS foram estabelecidas $^{(5)}$.

As atribuições desses agentes foram contempladas em conjunto com as da equipe multiprofissional de saúde, cabendo porém, ao gestor municipal, adequá-las às peculiaridades e necessidades locais de saúde. Entre as atribuições dos ACS, destacam-se o desenvolvimento de ações de integração entre a equipe de saúde e a população adscrita, a atuação junto às famílias cadastradas, a realização de atividades educativas e a promoção de ações de saúde e de prevenção de doenças ${ }^{(1)}$.

A partir de 2002, frente à ameaça de expansão da dengue, entre outras doenças infecciosas, o Ministério da Saúde determinou que as ações de controle e prevenção dessa enfermidade fossem integradas às da atenção básica, o que envolveu desenvolver e implementar medidas para a prevenção e controle desse agravo utilizando a Estratégia Agente Comunitário de Saúde (EACS) e a ESF. Esse enfoque combinado constituiu uma das propostas do Plano Nacional de Controle da Dengue (PNCD) para promover, na comunidade, mudanças de hábito que contribuam para manter o ambiente doméstico livre do vetor da doença ${ }^{(6-7)}$.
Por sua complexidade, as atribuições dos ACS requerem planejamento e gerenciamento pelo enfermeiro da equipe de saúde. Torna-se, portanto, indispensável que os agentes recebam orientação e treinamento periódicos para cumprir suas atribuições ${ }^{(8)}$. O novo perfil de atuação do ACS demanda formas mais abrangentes e organizadas de aprendizagem, para o que os programas de capacitação devem adotar uma ação educativa crítica capaz de referenciar-se na realidade das práticas e nas transformações políticas, tecnológicas e científicas do campo da saúde, de modo a assegurar ao agente o domínio de conhecimentos e habilidades específicas para o desempenho de suas funções ${ }^{(9)}$.

Considerando a importância da inserção dos ACS junto às equipes da ESF e o seu papel de interlocutor frente à população de sua microárea, faz-se necessário que o seu desempenho esteja de acordo com as diretrizes estabelecidas pela Política Nacional de Atenção Básica. Sendo assim, este estudo teve por objetivo caracterizar a atuação dos agentes comunitários de saúde lotados nas Unidades Básicas de Saúde da Família (UBSF) do município de Três Lagoas, em Mato Grosso do Sul.

\section{MÉTODO}

Para este estudo quantitativo-descritivo, foram entrevistados 68 ACS dentre um universo de 108 vinculados às UBSF do município. Os critérios de inclusão foram: atuar como ACS por, no mínimo, seis meses e estar trabalhando no período de coleta de dados. A coleta ocorreu nas sete UBSF de Três Lagoas em junho e julho de 2010 e abrangeu 13 equipes. Os ACS foram entrevistados pelos pesquisadores no início do expediente, após assinatura do Termo de Consentimento Livre e Esclarecido.

As variáveis analisadas foram: características sociodemográficas dos agentes comunitários de saúde, tempo de atuação, capacitações realizadas, frequência das visitas domiciliares, conhecimento das atribuições, desenvolvimento de atividades pertinentes, ou não, às suas atribuições e atividades desenvolvidas para controle e prevenção da dengue.

Os dados alimentaram uma planilha do programa Excel e os resultados foram analisados por estatística descritiva com frequência absoluta e porcentagem, e discutidos a partir da literatura pertinente. O estudo foi aprovado pelo Comitê de Ética em Pesquisa com Seres Humanos da Universidade Federal de Mato Grosso do Sul sob Parecer n. 1.728. 


\section{RESULTADOS}

Constatou-se que os ACS eram predominantemente mulheres (63 - 93\%), na faixa etária de 40 a 49 anos (25 - 36,8\%), casados (38 - 55,9\%) e haviam completado o ensino médio (Tabela 1).

Quanto ao tempo de atuação como ACS, entre 32 $(47,1 \%)$ essa experiência era de cinco a dez anos; em 24 $(35,3 \%)$ de seis meses a um ano; em $10(14,7 \%)$ superior a 10 anos; e em dois (2,9\%) era de três a cinco anos. A maioria dos entrevistados (62\%) já havia realizado o curso introdutório. Destes, 15 (35,7\%) o haviam feito antes de completarem um ano de atuação; 15 (35,7\%) de um a três anos; $9(21,4 \%)$ de cinco a dez anos; e 3 $(7,2 \%)$ de três a cinco anos.

Constatou-se unanimidade na participação em pelo menos um curso de capacitação, predominando os te-

Tabela 1 - Características sociodemográficas dos agentes comunitários de saúde $(\mathrm{n}=68)$. Três Lagoas, 2010

\begin{tabular}{lcc}
\hline Variáveis & N & \% \\
\hline Idade (anos) & 16 & 23,5 \\
$18-29$ & 18 & 26,5 \\
$30-39$ & 25 & 36,8 \\
$40-49$ & 9 & 13,2 \\
$50-59$ & - & - \\
60 ou mais & & \\
Estado civil & 12 & 17,6 \\
Solteiro & 38 & 55,9 \\
Casado & - & - \\
Viúvo & 5 & 7,4 \\
Divorciado & 13 & 19,1 \\
União estável & & \\
Grau de escolaridade & 6 & 8,8 \\
Superior completo & 12 & 17,6 \\
Superior incompleto & 48 & 70,6 \\
Ensino médio completo & 1 & 1,5 \\
Ensino médio incompleto & 1 & 1,5 \\
Ensino fundamental completo & - \\
Ensino fundamental incompleto & - & - \\
\hline
\end{tabular}

mas 'DST-aids', 'hanseníase e tuberculose', 'vacinação' e 'doenças infecto-contagiosas' (Tabela 2).

Todos os entrevistados relataram conhecer suas atribuições junto à equipe de saúde da família. Entre as atividades, destacaram-se as visitas domiciliares (Tabela 3).

Do total, 32 ACS (47\%) mencionaram realizar atividades que consideravam não ser de sua competência (Tabela 4). Dentre estas, predominou a entrega de medicamento e receita médica em domicílio (12 - 37,5\%), seguida da entrega de encaminhamento médico em domicílio (11 - 34,4\%).

Todos os entrevistados afirmaram realizar atividades de prevenção e controle de dengue, apesar de 20 (29\%) considerarem que estas não fazem parte de suas atribuições. A visita domiciliar para identificação de focos de dengue foi a mais mencionada (63 - 92,6\%) (Tabela 5).

Tabela 2 - Cursos de capacitação frequentados por agentes comunitários de saúde $(\mathrm{n}=68)$. Três Lagoas, 2010

\begin{tabular}{lcc}
\hline Tema & $\mathbf{N}$ & $\mathbf{\%}$ \\
\hline DST-aids & 62 & 91,2 \\
Hanseníase e tuberculose & 59 & 86,8 \\
Vacinação & 33 & 48,5 \\
Doenças infecto-contagiosas & 29 & 42,6 \\
Hipertensão arterial e diabetes & 28 & 41,2 \\
Oncologia & 28 & 41,2 \\
Trabalho do ACS & 23 & 33,8 \\
Planejamento familiar & 16 & 23,5 \\
Assistência materno-infantil & 15 & 22,1 \\
Atenção ao idoso & 14 & 20,6 \\
Técnico de ACS - Etapa I & 13 & 19,1 \\
Álcool e drogas & 11 & 16,2 \\
Saúde mental & 10 & 14,7 \\
Saúde do adolescente & 6 & 8,8 \\
Assistência farmacêutica & 5 & 7,4 \\
Primeiros socorros & 5 & 7,4 \\
Saúde da criança & 3 & 4,4 \\
Tabagismo & 3 & 4,4 \\
Outros & 6 & 8,8 \\
\hline
\end{tabular}


Tabela 3 - Atividades que os agentes comunitários de saúde relatam realizar $(n=68)$. Três Lagoas, 2010

\begin{tabular}{lll}
\hline Atividades & $\mathbf{N}$ & $\mathbf{\%}$ \\
\hline Visita domiciliar & 68 & 100,0 \\
Acompanhamento de grupos de risco & 57 & 83,8 \\
Educação em saúde & 56 & 82,4 \\
Vigilância em saúde & 46 & 67,6 \\
Mediador entre a comunidade e a equipe & 26 & 38,2 \\
Orientações quanto ao uso adequado dos serviços de saúde & 15 & 22,1 \\
Desenvolvimento de trabalhos junto aos membros da equipe & 14 & 20,6 \\
Entrega de receitas e medicamentos em domicílio & 11 & 16,2 \\
Cadastramento das famílias & 10 & 14,7 \\
Entrega de encaminhamentos médicos em domicílio & 9 & 13,2 \\
Agendamento de consultas médicas & 7 & 10,3 \\
Atualização de cadastros & 4 & 5,9 \\
Atividades vinculadas às atribuições & 4 & 5,9 \\
Solicitação ao médico de receitas e encaminhamentos para pacientes & 3 & 4,4 \\
Outras & 2 & 2,9 \\
\hline
\end{tabular}

Tabela 4 - Atividades que os agentes comunitários de saúde desempenham sem as considerarem como parte de suas atribuições $(\mathrm{n}=68)$. Três Lagoas, 2010

\begin{tabular}{lcc}
\hline Atividades & $\mathbf{N}$ & $\mathbf{\%}$ \\
\hline Entrega de medicamentos e receitas em domicílio & 12 & 37,5 \\
Entrega de encaminhamentos médicos em domicílio & 11 & 34,4 \\
Desenvolvimento de atividades administrativas & 7 & 21,9 \\
Acompanhamento de pacientes em serviços de saúde & 4 & 12,5 \\
Fornecimento de apoio emocional e psicológico & 4 & 12,5 \\
Preenchimento de formulários de dengue & 4 & 12,5 \\
Agendamento de consultas médicas & 3 & 9,4 \\
Atividades de enfermagem & 3 & 9,4 \\
Visita domiciliar para identificação de focos da dengue & 3 & 9,4 \\
Desenvolvimento de atividades fora do período de trabalho & 2 & 6,3 \\
Solicitação ao médico de exames e encaminhamentos para pacientes & 2 & 6,3 \\
Desenvolvimento de atividades em área descoberta & 1 & 3,1 \\
Notificação de doenças & 1 & 3,1 \\
Outras & 1 & 3,1 \\
\hline
\end{tabular}

Tabela 5 - Atividades de controle e prevenção de dengue realizadas por agentes comunitários de saúde $(\mathrm{n}=68)$. Três Lagoas, 2010

\begin{tabular}{lcc}
\hline Atividades & $\mathbf{N}$ & $\mathbf{\%}$ \\
\hline Visita domiciliar para identificação de focos & 63 & 92,6 \\
Orientação quanto à forma de evitar e eliminar criadouros & 49 & 72,1 \\
Informação ao morador sobre a doença & 48 & 70,6 \\
Informação ao morador quanto à verificação de larvas ou mosquitos transmissores & 19 & 27,9 \\
Eliminação de criadouros & 18 & 26,5 \\
Comunicação ao supervisor quanto à existência de criadouros ou mosquitos para que & 8 & 11,8 \\
sejam tomadas providências & 3 & 4,4 \\
Preenchimento de formulários específicos da dengue & 2 & 2,9 \\
Encaminhamento dos casos suspeitos de dengue ao serviço de saúde & 2 & 2,9 \\
Participação em atividades educativas & 1 & 1,5 \\
Outras & &
\end{tabular}




\section{DISCUSSÃO}

A predominância de ACS do sexo feminino neste trabalho configura uma tendência encontrada em outros estudos que avaliaram a proporção de mulheres atuando na área de saúde. Essa tendência pode estar intimamente relacionada ao papel que a mulher desempenha perante a sociedade: o de cuidadora, à qual cabe a maior parcela de responsabilidade pela saúde e bem-estar dos membros da família ${ }^{(10)}$.

$\mathrm{O}$ atendimento às exigências de escolaridade entre os participantes revelou-se superior ao mínimo estabelecido pela Lei n. 11.350/2006, que exige a conclusão do ensino fundamental para o exercício da profissão ${ }^{(5)}$. Semelhantes resultados foram encontrados em dois municípios do mesmo estado, o que permite inferir a baixa oferta de trabalho mais especializado e de salários mais altos nessas localidades ${ }^{(11)}$.

Quanto ao tempo de atuação, constatou-se tendência de permanência na atividade, com baixa rotatividade desses trabalhadores em Três Lagoas. Achados similares foram obtidos na região metropolitana de Recife $^{(12)}$. O tempo de permanência do ACS no exercício da atividade é de extrema importância para seu desempenho, uma vez que este é construído por meio de suas práticas cotidianas ${ }^{(10)}$.

Embora a participação dos ACS no curso introdutório no início de suas atividades profissionais seja um aspecto positivo, recomenda-se que este seja frequentado em conjunto com os demais profissionais da equipe. Isso permitiria discussões sobre os princípios e diretrizes do SUS e do contexto da ESF, de modo a subsidiar a organização do processo de trabalho ${ }^{(13)}$. Recomenda-se que o curso introdutório seja ministrado até três meses após a implantação da ESF, cabendo à Secretaria Estadual de Saúde, em parceria com a Secretaria Municipal de Saúde, a responsabilidade por sua oferta em municípios com menos de 100 mil habitantes. Quando a população alcança ou supera esse número, a responsabilidade passa a ser da Secretaria Municipal de Saúde, em parceria com a gestão estadual ${ }^{(1)}$. A maioria dos entrevistados informou haver participado de capacitações, com predominância de temas ligados a doenças infectocontagiosas, resultados estes que coincidem com os obtidos no município de Mossoró, Rio Grande do Norte ${ }^{(14)}$.

No desempenho de suas atribuições, os ACS necessitam receber capacitações e treinamentos que os auxiliem a desenvolver competências e habilidades técnicas, a fim de que possam transmitir, de forma clara e objetiva, os conhecimentos exigidos em seu processo de trabalho ${ }^{(14)}$ os quais, por sua diversidade e complexidade, muitas vezes ultrapassam o campo da saúde propriamente dito $^{(13)}$.

Dentre as atividades desenvolvidas pelos ACS, a mais citada foi a visita domiciliar, fundamental para a ESF por envolver desde o cadastramento até o acompanhamento da evolução de pacientes no âmbito da atenção básica ${ }^{(15)}$. É por meio dessas visitas que os ACS acompanham famílias e indivíduos ${ }^{(12)}$. Atividade intrínseca à ESF, a visita domiciliar proporciona ao profissional de saúde, e particularmente ao ACS, a possibilidade de adentrar o espaço da família e identificar suas peculiaridades, com visão ampliada de suas condições reais de vida. Além disso, a atividade promove a interação com o profissional de saúde tanto em âmbito familiar quanto social, uma vez que facilita o conhecimento do cotidiano e da cultura, incluindo crenças e costumes da população adscrita e, por extensão, da realidade social ${ }^{(16)}$.

Cabe ao ACS atuar na identificação de problemas, fornecer orientações, encaminhar pacientes e acompanhar a realização de procedimentos voltados à prevenção, proteção, promoção, recuperação e reabilitação dos indivíduos sob sua responsabilidade. A literatura aponta, porém, que esse agente desenvolve, em seu cotidiano de trabalho, algumas atividades que não são preconizadas pelo Ministério da Saúde, descaracterizando seu papel ${ }^{(10)}$.

Tal situação foi também evidenciada no presente estudo, já que atividades como entrega de receita e medicamento em domicílio, entrega de encaminhamento médico em domicílio, agendamento de consulta médica e solicitação ao médico de receitas e encaminhamentos para pacientes não se enquadram, conforme estabelecido pelo Ministério da Saúde, nas atribuições dos ACS. O mesmo foi evidenciado em unidades de saúde de Campinas (São Paulo) onde os agentes desempenham atividades administrativas, em razão de deficiências de recursos humanos e desconhecimento de suas atribuições por outros profissionais da equipe ${ }^{(17)}$. Realidade semelhante foi também identificada em Porto Alegre (Rio Grande do Sul) onde 13\% dos ACS informaram realizar trabalhos burocráticos, como prestar atendimento na recepção, entregar fichas de consulta, agendar consultas para a comunidade e dispensar medicamentos ${ }^{(10)}$.

O presente estudo também revelou que algumas atividades desenvolvidas pelos ACS, embora não percebidas por estes como parte de suas atribuições, são de fato preconizadas pelo Ministério da Saúde. É o caso do preenchimento de formulários de dengue 
e da visita domiciliar para identificação de focos e notificação da doença ${ }^{(1)}$. Situações como as detectadas podem gerar sobrecarga no trabalho desse profissional, pois qualquer ação que deva ser desenvolvida com as famílias passa a ser de sua responsabilidade. Assim, as ambiguidades e a polêmica que cercam sua figura persistem, tornando o ACS refém de atividades alheias a suas atribuições ${ }^{(18-19)}$.

Com relação às atividades de controle e prevenção da dengue, a Portaria n. 44/2002 define as atribuições dos ACS, considerando a importância de sua atuação na sua prevenção e controle ${ }^{(20)}$. A inserção dessas ações no cotidiano de trabalho desse profissional, particularmente na identificação de focos do vetor durante a visita domiciliar, permite os ACS atuar como multiplicador de informações importantes para a saúde da população adscrita, dado seu conhecimento sobre a realidade da comunidade, uma vez que nela reside. Isso permite que se fortaleçam os elos entre esta e o serviço de saúde, além de proporcionar melhorias nas relações de confiança, além de estimular o exercício da cidadania pela comunidade, com efeitos positivos na redução do risco de transmissão de dengue ${ }^{(7)}$.

Em estudo realizado no interior da Bahia constatou-se que os ACS têm visão limitada de suas atribuições, o que corrobora com os achados da presente pesquisa. Essa limitação pode estar relacionada à frágil formação, fundamentada principalmente no modelo biomédico. Faz-se necessário humanizar a formação, assim como promover reflexões sobre os princípios do Sistema Único de Saúde ${ }^{(21)}$.

\section{CONCLUSÃO}

O estudo revelou que os ACS apresentam fragilidades no reconhecimento de suas atribuições, que consistem em atividades básicas, como a visita domiciliar, a educação em saúde e o controle e prevenção da dengue, além de outras que lhe são alheias, tais como a entrega de receitas, medicamentos e encaminhamentos médicos em domicílio e o exercício de atividades administrativas na UBSF.

Torna-se necessário promover iniciativas que favoreçam a ampliação de investimentos em educação permanente e acompanhamento do trabalho realizado, a fim de se evitar descaracterização de seu papel, bem como prejuízos a seu cotidiano de trabalho, o que comprometeria a qualidade da assistência que esse profissional presta à população.

\section{REFERÊNCIAS}

1. Ministério da Saúde (BR). Portaria n. 2.488, de 21 de outubro de 2011. Aprova a Política Nacional de Atenção Básica, estabelecendo a revisão de diretrizes e normas para a organização da atenção básica para o Programa Saúde da Família e Programa de Agentes Comunitários de Saúde. Brasil SUS, [Internet] 24 out 2011 [acesso em 10 dez 2011]. Disponível: http://brasilsus.com.br/ legislacoes/gm/110154-2488.html.

2. Santana JCB, Vasconcelos AL, Martins CV, Soares JM, Dutra BS. Agente comunitário de saúde: percepções na estratégia da saúde da família. Cogitare enferm. [Internet] 2009;14(4) [acesso em $10 \mathrm{dez} 2011$ ]. Disponível: http://ojs.c3sl.ufpr.br/ojs2/index.php/ cogitare/article/view/16377.

3. Santos KT, Moimaz SAS, Arcieri RM, Carvalho ML. Agentes comunitários de saúde: perfil adequado à realidade do programa de saúde da família? Ciênc. saúde colet. [Internet] 2011;16(Suppl 1) [acesso em 15 jan 2012 ]. Disponível: http://dx.doi.org/10.1590/S141381232011000700035 .

4. Brasil. Lei n. 10.507, de 10 de julho de 2002. Cria a profissão de agente comunitário de saúde, e dá outras providências. Diário Oficial da República Federativa do Brasil, Brasília, 11 jul. 2002. Seção 1.

5. Brasil. Lei n. 11.350, de 5 de outubro de 2006. Regulamenta o $\S 5^{\circ}$ do art. 198 da Constituição, dispõe sobre o aproveitamento de pessoal amparado pelo parágrafo único do art. $2^{\circ}$ da Emenda Constitucional no 51, de 14 de fevereiro de 2006, e dá outras providências. Diário Oficial da República Federativa do Brasil, Brasília, 06 out. 2006. Seção 1.

6. Ministério da Saúde (BR). Fundação Nacional de Saúde. Programa Nacional de Controle da Dengue. Brasília: Ministério da Saúde; 2002.

7. Chiaravallotti Neto F, Barbosa AAC, Cesarino MB, Mondini A, Ferraz AA, Dibo MR, et al. Controle de dengue em uma área urbana do Brasil: avaliação do impacto do programa saúde da família com relação ao programa tradicional de controle. Cad. Saúde Pública [Internet] 2006;22(5) [acesso em $10 \mathrm{dez} 2011$ ]. Disponível: http://www.scielo.br/scielo.php?script=sci arttext\&pid=S0102-311X2006000500011\&lng=en\&n rm=iso>. ISSN 0102-311X. http://dx.doi.org/10.1590/ S0102-311X2006000500011.

8. Silva MJ, Rodrigues RM. O agente comunitário de saúde no processo de municipalização. Rev. Eletr. Enf.

Cogitare Enferm. 2012 Out/Dez; 17(4):635-41 
[Internet] 2000;2(1) [acesso em 14 set 2009]. Disponível: http://www.fen.ufg.br/revista/revista2_1/Agente.html.

9. Silva JA, Dalmaso ASW. O agente comunitário de saúde e suas atribuições: os desafios para os processos de formação de recursos humanos em saúde. Interface - Comunic., Saude, Educ. [Internet] 2002;6(10) [acesso em 14 set 2009]. Disponível: http://www. scielo.br/scielo.php?script=sci_arttext\&pid=S1414$32832002000100007 \& \operatorname{lng}=\mathrm{en} \& \mathrm{nrm}=\mathrm{iso}>$. ISSN 1414-3283. http://dx.doi.org/10.1590/S141432832002000100007.

10. Ferraz L, Aerts DRGC. O cotidiano de trabalho do agente comunitário de saúde no PSF em Porto Alegre. Ciênc. saúde colet. 2005;10(2):347-55.

11. Cazola LHO. Atuação do agente comunitário de saúde na Estratégia Saúde da Família e no Programa de Controle da Dengue: experiências de dois municípios brasileiros de pequeno porte [tese]. Campo Grande (MS): Universidade Federal de Mato Grosso do Sul; 2011.

12. Nascimento CMB. Análise do cumprimento das práticas dos agentes comunitários de saúde em municípios da região metropolitana do Recife [dissertação]. Recife: Centro de Pesquisas Aggeu Magalhães, Fundação Oswaldo Cruz; 2008.

13. Bornstein VJ, Stotz EN. Concepções que integram a formação e o processo de trabalho dos agentes comunitários de saúde: uma revisão da literatura. Ciênc. saúde colet. 2008;13(1):259-68.

14. Espínola FDS, Costa CC. Agentes comunitários de saúde do PACS e PSF: uma análise de sua vivência profissional. Rev. Odontol. Univ. São Paulo. 2006;18(1):43-51.

15. Barros DF, Barbieri AR, Ivo ML, Silva MG. O contexto da formação dos agentes comunitários de saúde. Texto Contexto Enferm. [Internet] 2010;19(1) [acesso em 10 dez 2011]. Disponível: http://www.scielo.br/pdf/tce/ v19n1/v19n1a09.pdf

16. Drulla AG, Alexandre AMC, Rubel FI, Mazza VA. A visita domiciliar como ferramenta ao cuidar familiar. Cogitare enferm. [Internet] 2009;14(4) [acesso em 10 dez 2011]. Disponível: http://ojs.c3sl.ufpr.br/ojs2/index. php/cogitare/article/view/16380

17. Nascimento EPL, Correa CRS. O agente comunitário de saúde: formação, inserção e prática. Cad Saúde Publica. [Internet] 2008;24(6) [acesso em $10 \mathrm{dez} 2011$ ]. Disponível: http://www.scielo.br/pdf/csp/v24n6/11.pdf
18. Nogueira RP. O trabalho do agente comunitário de saúde: entre a dimensão técnica "universalista" e a dimensão social "comunitarista". Interface - Comunic., Saude, Educ. [Internet] 2002;6(10) [acesso em 14 set 2009]. Disponível: http://www.scielo.br/scielo.php?script=sci_ arttext\&pid=S1413-81232008000100029\&lng=en\&n $\mathrm{rm}=\mathrm{iso}>$. ISSN 1413-8123. http://dx.doi.org/10.1590/ S1413-81232008000100029.

19. Tomaz JBC. O agente comunitário de saúde não deve ser um “super-herói”. Interface - Comunic., Saude, Educ. 2002;6(10):84-7.

20. Ministério da Saúde (BR). Portaria n. 44, de 3 de janeiro de 2002. Define as atribuições dos agentes comunitários de saúde no controle e prevenção da malária e da dengue. Diário Oficial da República Federativa do Brasil, Brasília; 08 jan. 2002. Seção1.

21. Rosa SR, Cavicchioli MGS, Bretãs ACP. O significado que o agente comunitário de saúde atribui ao seu trabalho no processo de construção do Sistema Único de Saúde. Acta Paul. Enferm. 2004;17(3):255-61. 